\title{
LAS SERIES ALCALINAS DEL PLIOCENO DE COSTA RICA: DISTRIBUCIÓN ESPACIAL Y RELACIÓN CON UNA FUENTE MANTÉLICA TIPO OIB
}

\author{
Esteban Gazel \\ Escuela Centroamericana de Geología, Universidad de Costa Rica. \\ egazel@geologia.ucr.ac.cr
}

(Recibido 1/3/04; aceptado 21/7/04)

\begin{abstract}
Pliocene alkaline magmatic rocks are reported in the costarican back-arc and paleo-arc (Aguacate Mountains). Teschenites and basanites from Guayacan Formation, are relative high in $\mathrm{P}_{2} \mathrm{O}_{5}, \mathrm{TiO}_{2}, \mathrm{Cr}, \mathrm{La}$ and Ni than basanites and alkaline basalts from La Garita Formation in the paleo-arc, with relative high $\mathrm{Al}_{2} \mathrm{O}_{3}$ and $\mathrm{Ba}$. $\mathrm{La} / \mathrm{Yb}$ and $\mathrm{K} / \mathrm{Ba}$ ratios evidence an OIB (ocean island basalt) mantelic source. Spider diagrams confirms that source, and $\mathrm{Ba} / \mathrm{La}$ ratios evidence that was subduction modificated. $\mathrm{Sr}^{87} / \mathrm{Sr}^{86}$ and $\mathrm{Nd}^{134} / \mathrm{Nd}^{144}$ ratios from Guayacan Formation related the OIB source with a Galapagos Hot Spot trace, the origin of the OIB source can be interpreted as the remelting of a Galapagos modified mantle or by direct Galapagos Plume enrichment. Local extention poceses, related with trans-tensional fault system in the central part of Costa Rica facilitated the magma raise.
\end{abstract}

Keywords: Alkaline series, OIB source, Pliocene, Geochemistry, Petrography, Costa Rica.

RESUMEN: En Costa Rica, se encuentran rocas magmáticas alcalinas del Plioceno, tanto en el transarco y en el paleoarco (montes del Aguacate). La Formación Guayacán en el trasarco, incluye la fase hipoabisal (teschenitas) y a la fase efusiva (basanitas), esta unidad presenta valores más altos de $\mathrm{P}_{2} \mathrm{O}_{5}, \mathrm{TiO}_{2}, \mathrm{Cr}$, La y Ni que la Formación La Garita en el paleoarco, la cual agrupa basanitas y basaltos alcalinos; esta última se caracteriza por presentar valores mayores de $\mathrm{Al}_{2} \mathrm{O}_{3}$. Las razones $\mathrm{La} / \mathrm{Yb}$ y K/Ba de estas dos formaciones corresponden con valores típicos para rocas alcalinas de tendencia OIB ocean island basalt. Los diagramas spider realizados confirman la fuente OIB y las razones $\mathrm{Ba} / \mathrm{La}$ indican que esta fuente se modificó con la subducción. Las razones isotópicas $\mathrm{Sr}^{87} / \mathrm{Sr}^{86}$ y Nd ${ }^{134} / \mathrm{Nd}^{144}$ de la Formación Guayacán se encuentran dentro del rango del punto caliente de las Galápagos. Por lo tanto esta fuente posiblemente se originó a partir de fusión parcial de un manto modificado en el punto caliente de las Galápagos o por el enriquecimiento directo por la pluma de las Galápagos. Procesos de extensión locales relacionadas con los sistemas de fallas transcurrentes que atraviesan el centro de Costa Rica facilitaron el ascenso de los magmas.

Palabras clave: Series alcalinas, Fuente OIB, Plioceno, Geoquímica, Petrografia, Costa Rica. 


\section{INTRODUCCIÓN}

Costa Rica se encuentra en el borde convergente de las placas del Coco y Caribe (Fig. 1). En la parte central se encuentran sistemas de fallas transcurrentes en una zona de aproximadamente $50 \mathrm{~km}$ de ancho. Astorga, et al. (1991) menciona que esta zona de deformación divide a Costa Rica en dos segmentos desde el Eoceno Superior/Oligoceno y la denomina Sistema de Falla Transcurrente de Costa Rica, el cual se caracteriza por generar cuencas tipo "pull-apart". Por otra parte Montero (2001) con base en estudios neotectónicos y sismológicos la denomina Cinturón Deformado del Centro de Costa Rica (CDCCR). Este sistema de fallas termina en el Cinturón deformado del Norte de Panamá (CDNP), donde predominan fallas inversas (Montero, 2001).

La cordillera volcánica Central es parte del arco magmático Cuaternario centroamericano, que se extiende desde la frontera MéxicoGuatemala hasta el complejo Irazú-Turrialba en Costa Rica. Este arco es el producto de la subducción de la placa del Coco bajo la del Caribe y sus características varían de acuerdo con las propiedades estructurales y tectónicas de la región (Carr et al., 1990). Esta cordillera presenta una longitud de $80 \mathrm{~km}$ y está conformada por cinco macizos volcánicos complejos; del NW al SE:
Platanar - Porvenir, Poás, Barva, Irazú y Turrialba. Todos presentan una composición mineralógica muy similar: andesitas basálticas y andesitas.

Durante el Plioceno el eje del arco magmático (montes del Aguacate) se encontraba más hacia el suroeste del arco actual, posiblemente respondiendo a un ángulo fuerte de subducción. Las rocas volcánicas de este paleoarco presentan una transición entre las series toleíticas y calcoalcalinas (Kussmaul et al, 1994) aunque también se encuentran rocas de tendencia alcalina subordinadas (Tournon, 1972). Para la misma época rocas volcánicas alcalinas se presentan con una mayor extensión en el trasarco Caribe. Estas rocas presentan razones $\mathrm{Ba} / \mathrm{La}(13-19)$ y $\mathrm{Nb} / \mathrm{Zr}$ $(0,17-0,46)$, que indican que se originaron a partir de una fuente mantélica enriquecida tipo OIB (Abratis \& Wörner, 2001).

Las rocas volcánicas recientes de Costa Rica se diferencian del resto de Centroamérica por presentar una influencia limitada de los fluidos del slab en la generación de los magmas (razones $\mathrm{B} / \mathrm{La}<0,5)$ y las razones isotópicas $\mathrm{Sr}$ ${ }^{87} / \mathrm{Sr}^{86}, \mathrm{Nd}^{134} / \mathrm{Nd}^{144}, \mathrm{~Pb}^{207} / \mathrm{Pb}^{206}$ y $\mathrm{Pb}^{208} / \mathrm{Pb}^{204}$ muestran que los magmas de la parte central de Costa Rica se originan en un fuente mantélica tipo OIB, con influencia de la pluma del punto Caliente de las Galápagos (Feigenson et al., 2004). Otra evidencia de la baja fusión parcial de un manto enriquecido es que las lavas con las

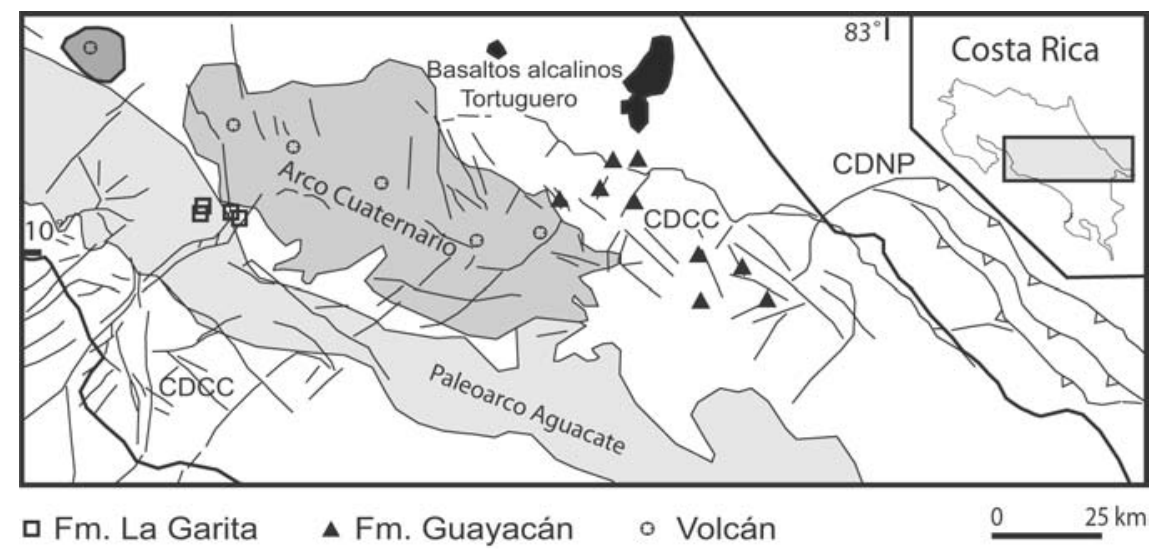

Fig. 1: Ubicación tectónica y espacial de las rocas alcalinas del Plioceno. CDCC: Cinturón Deformado del Centro de Costa Rica, CDNP: Cinturón Deformado del Norte de Panamá. Datos estructurales de Montero (2001) y Astorga et al. (1991). 
razones $\mathrm{Ba} / \mathrm{La}$ entre 20 y 40 presentan bajos $\mathrm{Be}^{10} / \mathrm{Be}^{9}$ (Leeman \& Carr, 1995). Esta misma fuente es reportada por Reagan \& Gill (1989) para lavas con alto contenido en $\mathrm{Nb}$ del volcán Turrialba y por Alvarado (1993) para lavas del volcán Irazú.

El modelo tectónico clásico, que relaciona a las rocas volcánicas alcalinas con la zona de sismicidad profunda en el trasarco, no es suficiente para explicar la distribución espacial de estas rocas en la región Caribe de Nicaragua y en Costa Rica. Abratis \& Wörner (2001) proponen que la subducción de la dorsal del Coco ha funcionado como una ventana en el slab, que ha permitido el ascenso de magmas alcalinos originados en una fuente tipo OIB. Tradicionalmente ha existido una tendencia de relacionar este magmatismo con límites de placa incipientes. Sin embargo, también puede originarse como consecuencia de colisiones continentales (graben del Rhin), vulcanismo transarco (islas Sándwich, Lau y Marianas) o relacionado con una zona de subducción profunda (Andes, Japón, Izu y el norte de Sulawesi). Los magmas basálticos presentes en estos ambientes tectónicos son predominantemente alcalinos (basaltos alcalinos, basanitas, nefelinitas, leucititas), aunque ocasionalmente se encuentran magmas félsicos (Wilson, 1994). Según Connor \& Conway (2000), emisiones alcalinas se han reportado en campos volcánicos relacionados con fallas profundas transtensionales. En el caso del campo volcánico de San Francisco (EE.UU.) se encuentran series máficas que incluyen basanitas, basaltos olivínicos alcalinos, basaltos toleíticos y andesitas basálticas.

\section{SERIES ALCALINAS DEL PLIOCENO}

\section{Formación Guayacán}

Se localiza entre las poblaciones de Siquirres y Guayacán. Azembre \& Tournon (1977) mencionan la presencia de alrededor de 20 diques y sills de teschenitas (de 3 a 40 m de espesor) en los alrededores del río Reventazón. Estos cuerpos subvolcánicos presentan una paragénesis de clinopiroxeno, plagioclasa y analcima, acompañados en proporciones variables de olivino, barquevicita, biotita titanífera, nefelina, hauyna, zeolitas y magnetita.

Tournon (1984) menciona la presencia de basanitas en los alrededores de Guayacán con inclusiones de cumulitos de peridotita, que evidencian una fuente mantélica con poca diferenciación. Stack (1991) describe 27 coladas de lava con un espesor total de $256 \mathrm{~m}$. Estos flujos de lava varían de masivas a vesicular mostrando estructuras, brechosas y columnares. Presentan fenocristales de olivino idiomórficos y microfenocristales de piroxenos en una matriz de plagioclasa, piroxenos, olivino, opacos, analcima intersticial, zeolitas y hauyna ocasional. Según el diagrama TAS se clasifican como basanitas y basaltos alcalinos (Fig. 2). Dataciones radiométricas muestran edades de 5,2 Ma de los basaltos alcalinos de río Chirripó; 4,6 Ma de las basanitas de Guayacán (Bellón \& Tournon, 1978) y 4,5 Ma de las techenitas de la quebrada Terciopelo.

\section{Formación La Garita}

Corresponde con emisiones lávicas de tendencia alcalina. Se ubica en los alrededores de La Garita y San Ramón y las mejores exposiciones se encuentran en las canteras del Puente de La Garita y Alto La Cima. Petrográficamente se caracterizan por presentar fenocristales de olivino, clinopiroxeno titanífero (de hasta $1 \mathrm{~cm}$ ) y plagioclasa cálcica, también se encuentra, biotita, opacos y analcima intercial. Según el diagrama TAS se clasifican como basanitas, basaltos alcalinos y traquibasaltos (Fig 2). Dataciones radiométricas muestran una edad de 2,8 - 3,2 Ma (Bellón \& Tournon, 1978).

\section{GEOQUÍMICA}

Con el propósito de analizar los procesos que han intervenido en la génesis de estos magmas, se recopilaron los análisis químicos, publicados por Tournon (1972), Picher \& Weyl (1976), Azambre \& Tournon (1977), Robin \& 


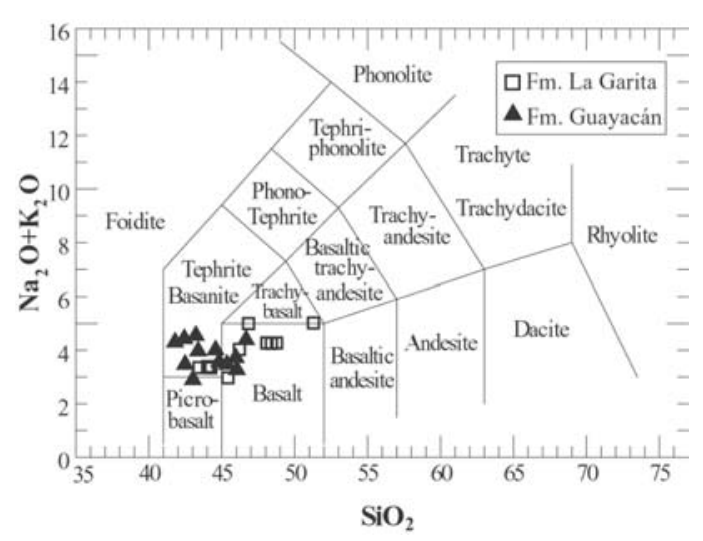

Fig. 2: Diagrama TAS (álcalis total - sílice), utilizado para la clasificación geoquímica de las rocas magmáticas alcalinas del Plioceno.

Tournon (1978), Laguna (1983), Weyland (1984), Stack (1991) y Malavassi (1991). Se incluyen tanto emisiones efusivas como las fases hipoabisales contemporáneas. Este banco de datos se encuentra disponible a solicitud.

Para diferenciar entre los campos alcalinos y sub-acalinos se utilizó el diagrama de Irvine \& Baragar (1971), el cual funciona bien para las series magmáticas alcalinas de Costa Rica (Fig. 3). Las rocas del Plioceno de Costa Rica se clasifican como basanitas, basaltos alcalinos y traquibasaltos (Fig. 2). De los datos recopilados se aprecia como existen dos poblaciones

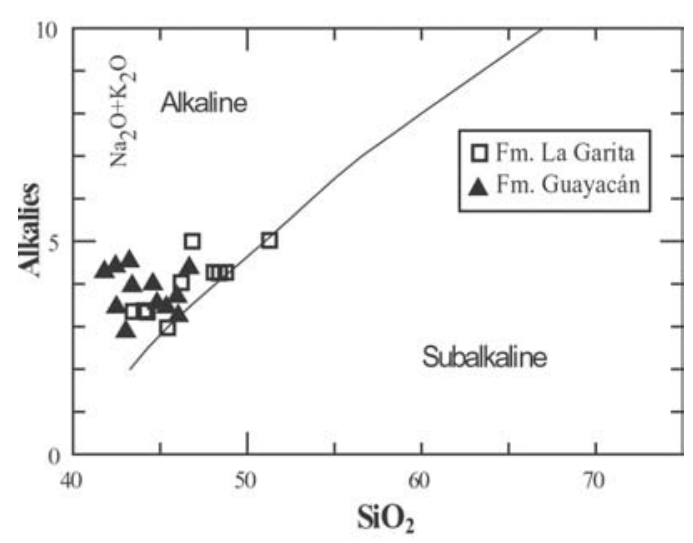

Fig. 3: Diagrama de Irving \& Baragar (1971) para diferenciar entre las series alcalinas y subalcalinas. bien definidas de datos: las rocas de la Formación Guayacán se caracteriza por presentar valores más altos de $\mathrm{P}_{2} \mathrm{O}_{5}, \mathrm{TiO}_{2}, \mathrm{Cr}$, Ni y La, que la Formación La Garita; por el contrario esta última presenta porcentajes mayores de $\mathrm{Al}_{2} \mathrm{O}_{3}$ (Fig. 4). Esto evidencia, que los procesos de cristalización de apatito y la entrada de Ti en minerales titanados (magnetita, clinopiroxenos u hornblenda) fue un proceso de mayor importancia en la génesis de las rocas alcalinas del trasarco. Las dos formaciones comparten valores $\mathrm{SiO}_{2} 41,8-49,0 \%$ y $\mathrm{TiO}_{2}>0,92 \%$.

Por otra parte la Formación Guayacán presenta razones $\mathrm{La} / \mathrm{Yb}(15,8-32,8), \mathrm{Ba} / \mathrm{La}$ $(10,9-19,5)$ y $\mathrm{K} / \mathrm{Ba}(8,9-22,8)$, mientras que la Formación La Garita presenta las razones Ba/La $(20,8-34,4)$ más altas y K/Ba $(6,8-15,0)$ más bajas la formación anterior. Las razones $\mathrm{La} / \mathrm{Yb}$ y K/Ba corresponden con valores típicos para rocas alcalinas de tendencia OIB (Wilson, 1994), originadas por un bajo grado de fusión parcial, no obstante las razones $\mathrm{Ba} / \mathrm{La}$ son cercanas a 10 en un OIB (Juteau \& Mauri, 1997), lo que indica que esta fuente se modificó por los fluidos del slab.

Los diagramas spider muestran una tendencia OIB (Fig. 5) puesto que al graficar estas rocas contra un MORB (Fig. 5) se obtiene un grafico que evidencia un magma enriquecido en HFS y LILES, por otro lado al graficar contra un OIB se logran valores cercanos a la unidad.

De acuerdo con los modelos de reservorios mantélicos propuestos por Zinder \& Hart (1986), las rocas alcalinas del Plioceno de la Formación Guayacán se acercan al Prevalent Mantle (Fig. 6) el cual es típico para fuentes tipo OIB y E-MORB presentes en puntos calientes (Galápagos, Islandia y Hawai). Las razones isotópicas $\mathrm{Sr}^{87} / \mathrm{Sr}^{86}$ y $\mathrm{Nd}^{134} / \mathrm{Nd}^{144}$ se encuentran dentro del rango de los valores del punto Caliente de las Galápagos.

\section{MODELO TECTÓNICO}

La presencia de rocas alcalinas en Costa Rica se explicó originalmente como un vulcanismo extensivo trasarco (Azembre \& Tournon, 

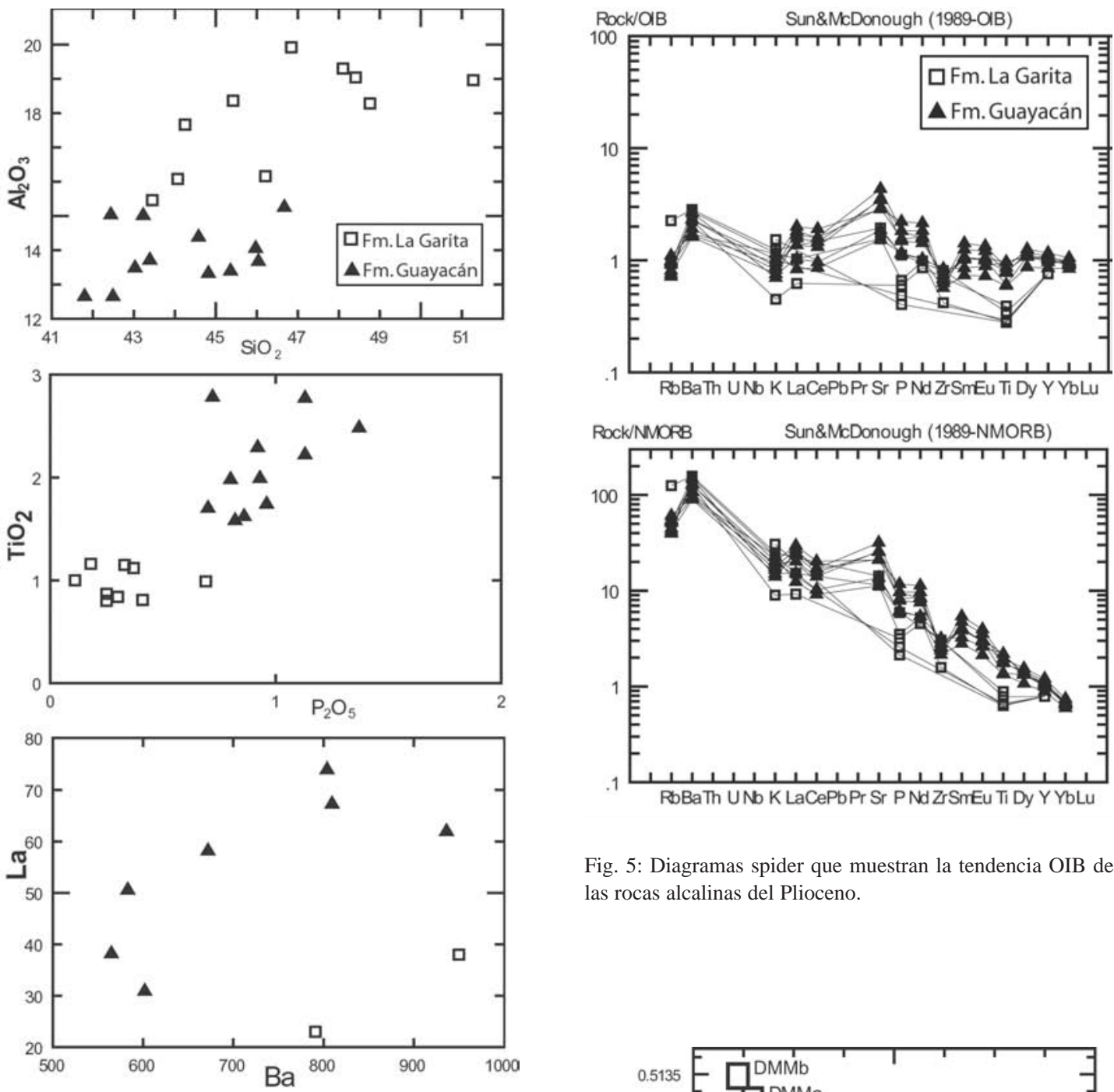

Fig. 5: Diagramas spider que muestran la tendencia OIB de las rocas alcalinas del Plioceno.

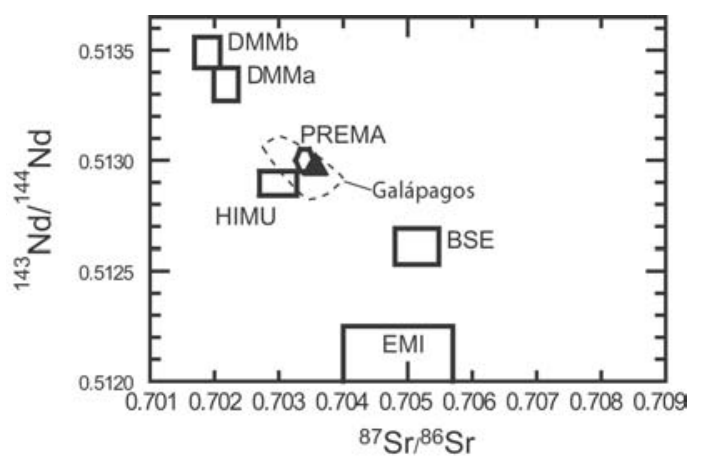

Fig. 6: Reservorios mantélicos de Zinder \& Hart (1986). DMM: manto deficiente, PREMA: manto prevalente, HIMU: manto con una razón $\mathrm{U} / \mathrm{Pb}$ alta, BSE: composición silícica

Fig. 4: Diagramas de variación de elementos mayores y trazas de la Fm. Guayacán y la Fm. La Garita. 
1977). Este modelo limita la posibilidad de emisiones alcalina al trasarco y por lo tanto no explica la presencia de estas rocas en el antearco.

Como se puede ver en la figura 1, las rocas alcalinas del Plioceno se alinean siguiendo el Cinturón Deformado del Centro de Costa Rica. El cambio de rumbo de estas fallas podría generar zonas de extensión locales, que incluyen cuencas tipo pull-apart, donde se ha facilitado el ascenso de magmas originados en una fuente mantélica tipo OIB (Fig. 7). Un proceso similar ocurre actualmente en el volcán Turrialba, el cual se encuentra $10 \mathrm{~km}$ atrás del arco y que se origina en medio de una cuenca de tracción (Linkimer, 2003). Los altos contenidos de Ti y de Nb, encontrados en basaltos de este volcán, surgieren que estos magmas se originan por medio de una coexistencia de una fuente mantélica OIB y una fuente de subducción (Reagan \& Gill, 1989). Lavas con alto $\mathrm{Nb}$ se han encontrado también en el volcán Poás (Malavassi, E., comun. pers. 2003).

\section{DISCUSIÓN Y CONCLUSIONES}

Las formaciones Guayacán en el trasarco y La Garita en el paleoarco del Aguacate se encuentran constituidas por rocas alcalinas que se clasifican químicamente como basanitas, basaltos alcalinos y traquibasaltos. La edad de estas rocas varía de los 5 a lo $2 \mathrm{Ma}$. Los análisis petrográficos y geoquímicos (altos y medios valores de $\mathrm{Cr}$, $\mathrm{Ni}$ y $\mathrm{MgO}$ ) muestran que estas rocas se originaron en una fuente mantélica primaria.

Las razones $\mathrm{La} / \mathrm{Yb}, \mathrm{K} / \mathrm{Ba}$ y los diagramas spider que esta fuente mantélica no se origina por medio de la fusión parcial de un manto tipo MORB, sino más bien responde a procesos tectónicos más complejos en los que interviene una fuente OIB enriquecida en elementos HFS y LILES. El origen de esta fuente mantélica puede explicarse mediante los siguientes modelos: 1) La corteza de Costa Rica se originó en el punto caliente de las Galápagos (Hauff, et al, 1999; Alvarado \& Denyer, 1998), de la misma manera el manto se modificó en este ambiente tectónico, por lo tanto la fusión parcial de un manto enriquecido podría explicar una fuente tipo OIB. 2) Es posible que el manto recibió y continue recibiendo un aporte directo de la pluma de las Galápagos por medio de fracturas o ventanas en el slab.

Procesos de extensión locales relacionadas con los sistemas de fallas transcurrentes que atraviesa el centro de Costa Rica facilitaron el ascenso de magmas alcalinos en regiones del paleoarco y el trasarco, donde no recibieron un aporte importante de la fuente de subducción y mantuvieron su naturaleza primaria. La presencia de la fuente OIB continua en las rocas volcánicas cuaternarias del la parte Central de Costa Rica.

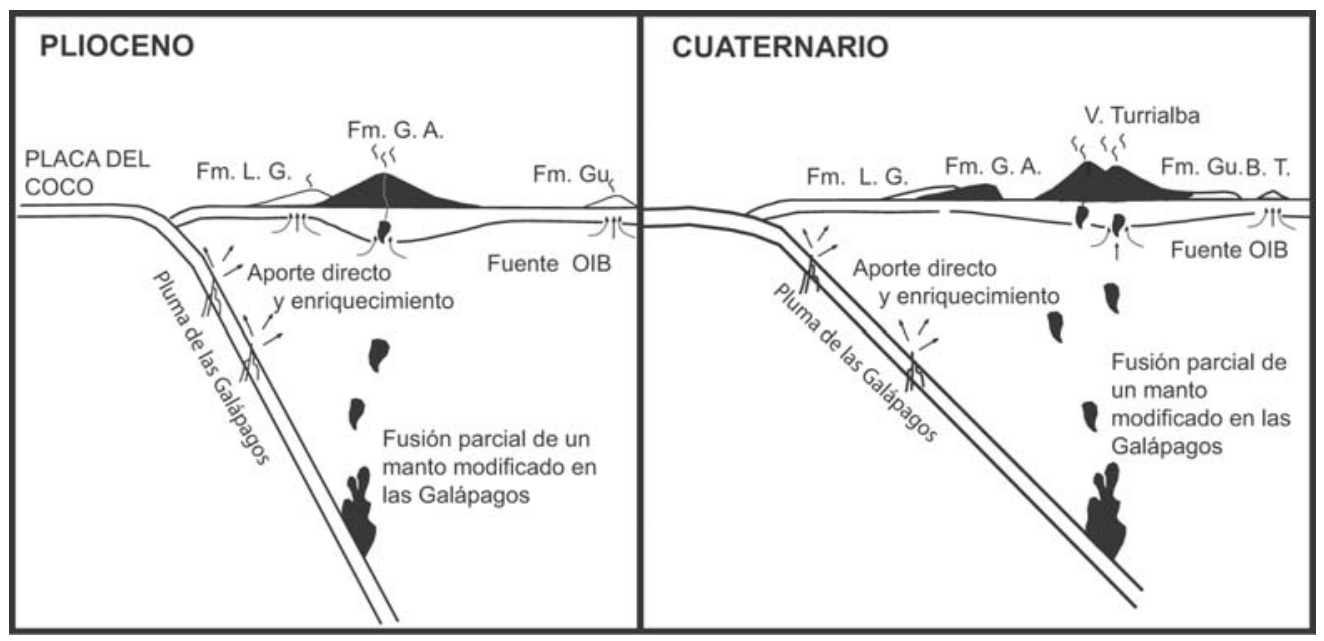

Fig. 7: Modelo tectónico simplificado, que explica la presencia de rocas magmáticas alcalinas en el paleoarco Aguacate y en la región trasarco. Fm. L.G., Formación La Garita; Fm. G.A.: Formación Grifo Alto; Fm. Gu.: Formación Guayacán, B.T: Basaltos alcalinos Tortuguero. 


\section{REFERENCIAS}

ABRATIS, M. \& WÖRNER, G., 2001: Ridge collisison, slap-window formation, and the flux of Pacific asthenosphere into the Caribbean realm. - Geol. Soc. Amer. 29(2): 127-130.

ALVARADO, G. E., 1993: Vulcanology and petrology of Irazu Vocano, Costa Rica. - 182 págs. Univ. Kiel, Alemania [Tesis Ph.D.].

ALVARADO, G. E. \& DENYER, P., 1998: Implications for the Caribbean Region of the high-Mg volcanic rocks in the Costa Rican ophiolite complexes: The case of the Tortugal, komatiitic-like suite. - Zbl. Geol. Palaont. Teil I, 3-6: 409-429.

ASTORGA, A., FERnÁNDEZ, J. A., BARBOZA, G., CAMPOS, L., OBANDO, J., AGUILAR, A., \& OBANDO, L.G., 1991: Cuencas Sedimentarías de Costa Rica: Evolución geodinámica y potencial de hidrocarburos. - Rev. Geol. Amér. Central, 13: 25-59.

AZEMBRE, B. \& TOURNON, J., 1977: Les intrusions basiques alcalines du Río Reventazón (Costa Rica). C.R. Som. Soc.Geol. France 2: 104-107.

BELLÓN, H. \& TOURNÓN, J., 1978: Contribution de la géochronométrie K-Ar a l'etude du magmatism de Costa Rica, Amérique Central. - Bul. Soc. Géol. France, 20(6): 955-959.

CARR, M.J., FEIGENSON, M.D., \& BENNETT, E.A., 1990: Incompatible element and isotopic evidence for tectonic control of source mixing and melt extraction along Central American arc. - Contrib. Min. Petrol. 105: 369-380.

CONNOR, B.C. \& CONWAY, M.F., (2000): Basaltic vocanic fields En: HARALDUR SIGURSON [ed.]: Enciclopedia of volcanoes-1417 pags. - A.P., San Siego, USA

FEIGENSON, M. D., CARR M. J., MAHARAJ, S. V., JULIANO, S. \& BOLGE, 1.1., 2004: Lead isotope composition of Cental American volcanoes: Incluence of the Galapagos plume. - Geochem, Geophys, Geosystems. <http://gcubed.magnet.fsu.edu/index.asp> [consulta: julio 2004]

HAUFF, F., HOERNLE, K., BOOGARD v.d., P., ALVARADO, G. E., \& GARBE-SHÖNBERG, D., 2000: Age and geochemistry of basaltic complexes in western Costa Rica: Contribution to the geotectonic evolution of Central America. - Geochem, Geophys, Geosystems. <http://gcubed.magnet.fsu.edu/index.asp> [consulta: agosto 2003]

IRVINE, T.N. \& BARAGAR, W.R.A., 1971: A guide to the chemical clasification of common volcanic rocks.Can.Earth Sci. 8: 523-548
JUTEAU, T. \& MAURY, R., 1997: The oceanic crust, from accreation to mantle recycling. - 390 págs. SpringerPraxis, Chichester UK.

KUSSMAUL, S., TOURNON, J., \& ALVARADO, G., 1994: Evolution of the Neogene to Quaternary igneous rocks of Costa Rica. - Profil, 7: 97-123.

LAGUNA, J., 1983: Petrographie, Petrologie und Tonmineralogie der Aguacate-Formation (Miozän-Pliozän), Costa Rica, Zentralamerika. - 163 págs. PhillippsUniversiät, Marburg [Tesis PhD.]

LEEMAN, W.P. \& CARR, M., 1995: Geochemical constraints on subduction processes in the Central American Volcanic Arc: Implications of boron geochemestry. - Geol. Soc. Amer. Special Paper 295: 57-73.

LINKIMER, L., 2003: Neotectonica del extremo oriental del Cinturón Deformado del Centro de Costa Rica - 103 págs. Univ. Costa Rica [Tesis Lic.].

MONTERO, W., 2001: Neotectónica de la región central de Costa Rica: frontera oeste de la microplaca de Panamá. - Rev. Geol. Amér. Central, 24: 29-56.

PICHER, H. \& WEYL, R., 1976: Magmatism and crustal evolution in Costa Rica (Central America). - Geol. Rundsch. 64: 457- 475.

REAGAN, M. K. \& GILL, J.G., 1989: Coexisting of calcoalcaline and higth niobium basalts from Turrialba volcano, Costa Rica: Implications for residual titanites in arc magma sources. - J. Geophys. Res. 94: 46914633.

ROBIN, C. \& TOURNON, J., 1978: Spatial relations of andesitic an alkaline provinces in Mexico and Central America. - Can. J. Earth. Sci. 15: 1633-1641.

STACK, C., 1991: Inverse modeling of alcaline lavas from Guayacan, Costa Rica. - 52 págs. State. Univ. New Jersey [Tesis MSc.].

TOURNON, J., 1972: Présence de basaltes alcalins récents au Costa Rica (Amerique Centrale). - Bull. Volcanol. 26 (1): 140-147.

WEYLAND, T., J., 1984: The petrology , chemestry, and evolution of the Aguacate Formation, Tilaran Cordillera and Aguate Mountains, Costa Rica, Central America. - 133 págs. Univ. North Carolina [Tesis $\mathrm{PhD}$.].

WILSON, M., 1994: Igneous petrogenesis: A global tectonic approach. - 466 págs. Chapman and Hall, London.

ZINDER, A. \& HART, S.R., 1986: Chemical geodynamics. Ann. Rev. Earth Planet. Sci. 14: 493-571 